\title{
Preface to the Special Issue: Additive Manufacturing
}

\author{
Xin $\operatorname{Lin}^{1}$
}

Published online: 18 January 2022

(c) The Chinese Society for Metals (CSM) and Springer-Verlag GmbH Germany, part of Springer Nature 2022

Additive manufacturing (AM) with particular processing characteristics shows excellent potential to innovate the manufacturing industry and the production mode. AM offers a considerable degree of design freedom and allows manufacturing of geometries that are not feasible by conventional manufacturing and is treated as a superb solution reducing lead time to market for new products. Moreover, the pointwise forming characteristics empower this technique to produce refined and above supernormal microstructures owing to rapid cooling and non-equilibrium solidification, creating more possibilities for material microstructure modification.

After years of investigating and testing in the laboratory environment, the AM technique has been gradually accepted and popularized by the manufacturing industry recently. Research activities in universities, government, and industrial laboratories, and the research focus in a large number of conferences and publications reflect the ever-increasing interest in AM. Therefore, Acta Metallurgica Sinica (English Letters) Editorial Committee and I jointly conceived this AM special issue, expecting to summarize and demonstrate the research status, highlights, and future developing direction of AM by selecting excellent research articles with the theme of AM.

This special issue on AM was designed to comprehensively present the wide range of topics to be addressed by the AM research community, such as the innovative structure design of the component, multi-scale modeling, special materials applicable to AM, heat and mass transfer during the process, microstructure and property characterization of the AM-built component, process optimization, etc. The above topics involve interdisciplinary subjects such as material science, engineering technology, structural mechanics,

Xin Lin

xlin@nwpu.edu.cn

1 State Key Laboratory of Solidification Processing, Northwestern Polytechnical University, Xi' an 710072, China and so on, which will attract the attention of scholars or engineers in a wide range of fields.

I would like to take this opportunity to thank the Acta Metallurgica Sinica (English Letters) Editorial Committee for providing an opportunity for researchers around the world to publish their latest and outstanding research results and provide them to the broader AM research community. I would also like to thank the paper's authors for taking time from their busy schedules and for their hard work in the writing, submitting, and revising processes. The data, results, and new knowledge presented by the authors help all our researchers better understand the process-structureperformance relationship in AM. Meanwhile, we also welcome readers to communicate with the editorial committee or authors, putting forward valuable opinions and jointly contributing to further innovation and upgrading of the AM technique.

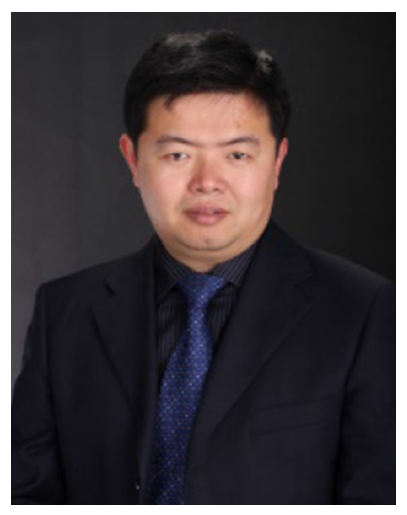

Xin Lin is Director of Key Laboratory of Metal High-Performance Additive Manufacturing and Innovative Design, Ministry of Industry and Information Technology (MIIT), China. His research areas include metal additive manufacturing (AM) and solidification. He holds 30 Chinese patents and has published three monographs and over 400 articles in journals, such as Acta Mater., Addit. Manuf. His research on metal AM has found commercial applications in aviation, aerospace, power, energy, and medical fields, and met the urgent needs for highperformance, light-weighting, high integration, and high precision fabrication in these high-tech fields. The Royal Society awarded him a Newton Fellowship in the UK in 2015. 